\title{
Early Intervention in Schizophrenia
}

\author{
Serafín Lemos*, Oscar Vallina** and M. Purificación Fernández** \\ *Universidad de Oviedo, España. **Hospital de Sierrallana, Torrelavega, España.
}

\begin{abstract}
The objective of this work is to report the current situation of some international programs of early intervention in psychosis, analyzing the criteria used for identifying persons at risk and the difficulties involved in defining risk in retrospective and prospective studies. There is a brief discussion of the objectives of the programs of early intervention in psychosis and the components of the protocol. Finally, the authors offer a short description of the project under way in a public health service in a region of northern Spain. Key words: primary indicated prevention, early intervention, schizophrenia.
\end{abstract}

\section{RESUMEN}

Intervención temprana en esquizofrenia. El objetivo de este trabajo es exponer la situación actual de algunos programas internacionales de intervención temprana en psicosis, analizando los criterios que se utilizan para la identificación de las personas de riesgo y las dificultades existentes para definir el riesgo en los estudios retrospectivos y prospectivos. Se describen brevemente los objetivos de los programas de intervención temprana en psicosis y los componentes del protocolo. Finalmente, se describe brevemente el proyecto que los autores han puesto en marcha en un servicio público de salud de un área del norte de España.

Palabras clave: prevención primaria indicada, intervención temprana, esquizofrenia.

Programs of early intervention in psychosis are based on the conviction that, in general, in order to achieve a full recovery from a disorder or illness, intervention should take place as early as possible.

In the case of psychoses, although the data are not yet conclusive, the impression emerging from research is that their clinical outcome is also influenced by early intervention (McGlashan, 1998). A psychosis that occurs in a young person (e.g., under 20) is quite likely to involve schizophrenic characteristics; if its onset can be delayed (e.g., until beyond age 30), it is likely to express itself with more benign clinical characteristics.

${ }^{1}$ Reprints may be obtained from the first author: Facultad de Psicología, Universidad de Oviedo, Plaza Feijóo, s/n, 33003 Oviedo, España. Email: slemos@correo.uniovi.es 
Thus, clinical experience suggests that the outcome of early-onset schizophrenia (in adolescence, for example) is significantly worse than if the schizophrenia begins in adulthood, perhaps because of biological and psychosocial factors (Rapoport et al., 1999).

Early intervention in psychosis, that is, when the disorder is still at a subsyndromic level (with symptoms below the critical threshold), is an example of what is called "indicated primary prevention" (Mrazek \& Haggerty, 1994). It is believed that the identification of people in this early stage of the disorder, followed by psychosocial and pharmacological intervention, can prevent a full-blown disorder.

Even so, how to detect those at risk early enough to initiate an intervention program, how to differentiate the risk of developing a psychosis from the predisposition to other, less serious mental pathologies, and to what extent early intervention succeeds in preventing the transition to psychosis in the predisposed person, are all still matters of debate. Nevertheless, it is clear that an intervention procedure with these objectives requires (a) adequate identification of those at risk, (b) a proper understanding of the characteristic symptoms and patterns of change in neurobiological and psychological processes of the pre-psychotic phase, and (c) developing types of intervention for this phase that succeed in improving the symptoms and the person's everyday functioning and preventing deterioration.

\section{EARLY DETECTION}

\section{Identification of prodromic signs}

It would seem reasonable to think that psychosis, just like depression or other disorders, is distributed continuously among the population (though possibly more asymmetrically, due to its lower prevalence), and that it can be identified at an early stage through certain characteristics or symptoms.

However, the assumption of a continuous distribution of psychosis has been interpreted from two different points of view, which gave rise to two predominant approaches in the study of predisposing factors: a retrospective, clinically-oriented approach, which attempts to find attenuated signs and symptoms, premonitory of the disorder, several years prior to its manifestation proper, and a prospective approach, aimed at identifying those considered to be high risk, due to the presence of certain traits or markers, or merely to genetic predisposition. These two approaches have been based, respectively, on the identification of prodromic signs and of premorbid traits or characteristics of people considered to be at risk.

The majority of recent research has been of a retrospective nature, since the concept of prodrome in psychosis refers to the reconstruction of the symptoms manifested in the period from the appearance of the first unusual experiences or appreciable symptoms up to the first acute psychotic crisis (Loebel et al., 1992; Yung \& McGorry, 1996); thus, this phase, which is generally non-specific and diffuse, is confirmed as prodromic when a recognized disorder does in fact subsequently emerge.

The concept of prodrome, however, is used to designate not only the symptoms 
preceding the pre-psychotic period of the first episode, but also a psychotic relapse; thus, while the prodromic symptoms of the first episode of a schizophrenic psychosis may last from a few weeks to several years (Beiser, Erickson, Fleming \& Iacono, 1993; Häfner et al., 1999; Loebel et al., 1992), the duration of relapse prodromes is generally a few hours to a few days (Yung \& McGorry, 1996).

Systematic studies of the prodromes of psychosis have focused on subjective symptoms and objective changes in behaviour, which include attention problems, apathy and lack of motivation, depression, sleep disorders, anxiety, social isolation, lack of confidence, deterioration in school or work performance, and anger or irritability (Yung \& McGorry, 1996).

Bearing in mind that comorbidity is, moreover, a highly frequent characteristic in the young population, even after the diagnosis of a predominant disorder, the task of recognizing the specific prodromic signs of the psychosis often becomes complicated.

Research, though, indicates that the psychopathological characteristics of the prodromic phase of psychosis are quite diverse and expressed with highly unspecific symptoms; however, there tends to be a succession from unspecific symptoms to negative symptoms and positive symptoms, which mark the onset of the acute expression. Thus, Häfner et al. found that only around $10 \%$ of schizophrenic psychosis cases began with positive symptoms, $20 \%$ with positive and negative symptoms simultaneously, and $70 \%$ with negative symptoms (and of these, half took two years or more to develop any positive symptoms (Häfner \& Maurer, 2001; Häfner et al., 1999).

Using the semi-structured Interview for Retrospective Assessment of the Onset of Schizophrenia (IRAOS), Häfner et al. assessed the social development, premorbid adaptation, prodromic signs, clinical symptoms and social incapacities of a large sample of patients with schizophrenia, by means of data from three sources: patients themselves, their close family, and medical documents (clinical history, etc.) (Häfner et al., 1992). The results indicate that among the 10 initial symptoms of the psychotic disorder, positive symptoms are normally absent, and that there is a predominance of affective symptoms (depressive mood, lack of self-confidence, worry, uneasiness and anxiety) and negative symptoms (thinking and concentration problems, lack of energy, slowness, poor work performance and social isolation) -that is, characteristics that reveal a lack of emotional response, or flat affect. The onset of the acute psychotic episode, on the other hand, is characterized by the emergence of positive symptoms, notable among which are ideas of reference, persecutory delusions and other delusions, followed by auditory hallucinations.

In sum, generally, the first appreciable symptoms are affective, and are followed by negative symptoms (between 2 and 5 years before the first admission); the positive symptoms appear in a subsequent phase (1 year before the first admission).

On basing the identification of the prodromic signs on retrospective analyses, we find two problems: on the one hand, the existence of possible recall deficits and memory distortions, and on the other, the difficulty of discriminating between non-specific early symptoms of psychosis and other antecedents and symptoms with other causes. For example, prodromic signs of schizophrenia may be similar to those of the initial phases of depression, so that it is generally difficult to conclude whether the first signs of 
change will lead to schizophrenic psychosis, to an anxiety disorder or to a depression, since the changes may go in the direction of either a state of isolation or one of hyperfunction (nervousness and unease). Thus, numerous studies attempting to describe the clinical characteristics of the prodromic phase of psychosis have shown scarce predictive power (McGorry, 1998).

Indeed, as the concept of prodrome is not applicable to prospective studies, McGorry et al. have preferred to use the term "high risk mental state" to define three syndromes that are supposedly premonitory of psychosis -even though the transition from them to psychosis is not considered inevitable (Yung, McGorry \& McFarlane, 1996). This approach is similar to that employed by other authors with regard to subclinical depressive states, as risk factors for depression (Pincus, Davis \& McQueen, 1999).

The studies by McGorry et al. in Australia (McGorry \& Jackson, 1999; Yung et al., 1998) and McGlashan et al. in New Haven (USA) (McGlashan, 1998; McGlashan, Miller, Woods et al., 2001) have permitted the combination of several predisposition factors and the definition of the following three categories of high risk mental state:

Group 1: People with attenuated positive symptoms (or below the clinical threshold), such as ideas of reference, strange beliefs, magical thoughts, perceptual alterations, paranoid ideation, and strange behaviour or appearance. Added to this list in other studies are attenuated and non-specific negative symptoms: depressive mood, social isolation, deterioration in functioning, deterioration in personal hygiene, loss of capacity for concentration, loss of motivation, sleep disorders and anxiety (Cornblatt, Lencz \& Obuchowski, 2002).

Group 2: People with transitory or intermittent psychotic symptoms (ideas of reference, magical thoughts, perceptual distortions, paranoid ideation or strange language), which are resolved spontaneously in one week.

Group 3: People displaying a combination of genetic risk (e.g., close relative of a person diagnosed with some schizophrenic spectrum disorder) and a significant deterioration in mental state or a change in level of functioning (considerable deterioration compared to the previous year).

Studies carried out in Australia, the United States and Norway have found that people fulfilling the criteria of any of these groups have a rate of transition to psychosis within one year of between $21 \%$ and $54 \%$; this probably means that they are patients in the prodromic phase, for whom, consequently, treatment would be advisable (McGlashan, Miller \& Woods, 2001). Thus, these high rates of transition to psychosis indicate that people fulfilling these criteria are not simply asymptomatic, or even genetically vulnerable, and that they are not in a premorbid phase, but that they are people on the edge of psychosis and will easily develop symptoms and incapacities. This normally leads them to seek professional help.

On the other hand, we cannot be sure that the three groups mentioned above are homogeneous, or that they have the same etiology; therefore, objections may be raised over their use in the design of a single, common program of early intervention and, in particular, with identical psychopharmacological treatment.

Another of the main drawbacks with prodromic studies, and especially with the 
three risk categories above, is that they almost certainly identify people with actual psychotic disorders (even though compensated), and not people at risk as such; it is possible that this may occur also with the attempt to identify people at risk using certain instruments designed for this purpose, such as the IRAOS interview already mentioned, or the SIPS (Structured Interview for Prodromal Syndromes) (McGlashan, Miller, Woods et al., 2001), in which prodromes are in fact psychotic symptoms on the threshold of a disorder.

In the view of McGlashan, Miller \& Woods (2001), however, the experience accumulated with the use of these identification criteria reveals that the number of people recruited is quite close to the rate of incidence of schizophrenia (that is, 1/ 10,000 per year), so that a higher recruitment may indicate that incorrect criteria are being used, and that, consequently, there is a risk of selecting many false positives. Thus, despite attempting to improve the risk markers and their predictive capacity, they feel that the current criteria confer a sufficient degree of accuracy in prediction for them to be ignored. In any case, future follow-up studies will throw more light on this issue.

Consequently, it can be concluded that the symptoms and deviations that precede the onset of schizophrenia and other psychoses are somewhat unspecific, so that it is advisable to carry out a stepwise process of identifying persons at risk. Some projects have used such an approach, among them the DEEP (Salokangas et al., 2001), applied in Finnish and North American universities, which starts out from the hypothesis that the onset of a psychosis is preceded by detectable pathological changes in the central nervous system. The pathophysiological mechanism may be extensive pruning of the cortico-basal interneural connections and those in the cerebral cortex, which would cause the CNS to lose capacity for maintaining integration in the cognitive and emotional functions and between them.

The neuropsychological alterations observed in attention, memory and the executive functions are thought to indicate deterioration in frontal and temporo-medial regions of the brain. The resulting deficits may be in large part independent of the clinical state, but on the other hand they would explain the functional deterioration in the social and occupational areas (work or school).

\section{Prospective studies}

Longitudinal studies on the predictor traits of psychosis tend to avoid retrospective methodology, which aims to identify prodromic signs, and generally involve prospective follow-up of the descendants of mothers with schizophrenia. These approaches make use of external behavioural assessments, such as parents' and teachers' reports, and monitor individual neuromotor, neuropsychological, physiological and, to some extent, morphological, parameters from an early age. Among such work are the well-known Copenhagen (Olin et al., 1998), New York (Cornblatt \& Erlenmeyer-Kimling, 1985; Squires-Wheeler, Friedman \& Erlenmeyer-Kimling, 1993) and Israel high risk studies (Marcus et al., 1987).

In these studies, high risk children that do not develop a psychosis are the ideal 
controls to be compared with those that actually manifest disorders. In the case that the two groups had similar family environments, the belief is that, in theory, it would be possible to identify more specific predictive factors.

The Copenhagen high risk project has stressed the importance of genetic risk (descendants of parents with schizophrenia), but also its interaction with obstetric complications. Nevertheless, bearing in mind that a high percentage of schizophrenics did not have parents with this disorder, the vast majority of potential cases of schizophrenia cannot be identified with the genetic criterion alone; thus, it is necessary to combine the genetic information with other premorbid indices to increase accuracy with regard to predictive factors (Carter \& Mednick, 2001). In this project, the premorbid indicators taken into consideration are the children's rearing environment, their behavior in school, and their personality.

The Copenhagen study reports that the risk of schizophrenic psychosis increased when children with high genetic risk remained in institutions for more than 6 months continuously during the first 10 years of life (reaching a rate of $25 \%$ of schizophrenia diagnosis in these cases, as against a rate of $12 \%$ in high risk children who grew up without such prolonged institutionalization). The effect was independent of the diagnosis of the father or the clinical seriousness of the mother. Identical results were obtained in the high risk studies in Israel (Marcus et al., 1987; Mirsky, Kugelmass, Ingraham, Frenkel \& Nathan, 1995). Secondly, deviant school behaviour was also an important precursor (more active and disruptive behaviour in boys and more nervousness in girls). This pattern of behaviour and the sexual differences were also described in the New York high risk project (Watt, Anthony, Wynne \& Rolf, 1984). Finally, high values on the scales assessing depression, anxiety, schizophrenia and social introversion, from the MMPI, (as well as a high score on the F scale) were also important predictors.

The sensitivity of these indices (that is, the proportion of true positives) varied slightly, personality (according to the MMPI) being the most sensitive index (60\%), followed by the school scales (47\%) and the institutionalization measure (35\%). As regards specificity (that is, the proportion of true negatives), the index of the rearing environment was the most specific (85\%), followed by the indices of school behaviour $(79 \%)$ and of personality $(66 \%)$. However, the percentage of risk subjects, identified by means of any of these three indices separately, is low (as compared to the percentage of normal children), and this is almost certainly due to the fact that a single variable has low discriminatory power. It is for this reason that it is recommended to combine several different theoretically important variables in a multivariate design.

In recent analyses, Carter and Mednick tried to maximize predictive power by combining variables showing predictive power in one equation, which includes genetic risk, birth complications, physiological skin conductance measures, rearing environment, neurocognitive variables, personality and school behaviour. Of all of these variables, the combination of the following five was that which significantly predicted the schizophrenia diagnosis in young people with high risk: genetic risk because of father diagnosed with a schizophrenic spectrum disorder, long periods of institutionalization prior to age 10, poor relations with the mother, unusual personality traits (according to the F scale of the MMPI) and problematic school behaviour. Together, these variables 
explained $23 \%$ of the variance in diagnostic outcome.

These predictor variables proved to be more specific for schizophrenia than for other psychoses, since those who developed a non-schizophrenic psychosis showed, in general, less deviation during adolescence.

In other prospective studies carried out with a cohort of British children, Jones et al. reported that people who developed a schizophrenic psychosis mastered certain skills (such as walking) proportionally later, by comparison with normal children, and that they had more problems with language and poorer school performance, preferred playing alone and showed less self-confidence; moreover, at age 15 their teachers considered them more anxious in social situations. Even so, it is also pointed out that these characteristics had low specificity (Jones, Rodgers, Murrray \& Marmot, 1994).

A final reflection on the risk factors emerging from these prospective studies concerns the fact that, supposing there is a multifactorial etiology of psychosis, the identification of the relevant variables for predicting the risk and the distribution of predisposition would depend on the degree to which the causes interact, their prevalence, and the degree to which their effect sizes differ. In the case of there being large differences in the effect size, with one or two causes that are very unusual but extremely powerful, as is the case of genetic predisposition, the effect of other causes, more prevalent but weak, would be overshadowed; thus, the appearance of the distribution of predisposition to psychosis would also be less continuous and more quasi-continuous, as in fact seems to be the case.

\section{EARLY INTERVENTION PROGRAMS}

Up to the present, there have been no premorbid intervention programs that are well-structured and subjected to research; rather, programs have been designed aimed at the identification of prodromic symptomatology and early intervention on it. It is still an open question, therefore, as to whether intervention in adolescence can prevent or reduce the morbidity of psychoses that may take effect many years later.

It would seem clear that psychological interventions can improve social skills or other resources for coping with stress; it is also clear that psychopharmacological interventions can reduce certain types of cognitive dysfunctions. It can further be assumed that seeing a clinical psychologist in adolescence makes it more probable that an adult with disorders will seek help when it is needed. Nevertheless, these benefits are mere speculations that require empirical verification.

Currently, clinicians and researchers are devoting most of their efforts to treating both the initial symptoms of the first psychotic episode and the symptoms that precede it; nevertheless, such responses cannot really be considered as preventive interventions, since the process of the disorder has normally already begun.

Although current treatments for schizophrenia, which include antipsychotic medication, psychoeducation and family intervention, have shown themselves to be effective (Vallina Fernández \& Lemos Giráldez, 2001), they can be considered as palliative, and must be maintained indefinitely if it is intended to reduce the probability of symptomatic relapses or functional deterioration. 
In recent years, various studies have stressed that early treatment of schizophrenia correlates with better clinical outcomes; thus, what has come to be called "duration of untreated psychosis" (DUP) has been the object of detailed analysis (Friis et al., 2003), and its shortening has become a therapeutic objective, on the basis of research appearing to demonstrate that it is a good predictor of clinical outcome (in particular, of the probability of relapses and functional deterioration) and of the medical cost of each case (Moscarelli, 1994).

The relationship between DUP and the response to treatment or clinical outcome is, however, merely correlational, and subject to diverse interpretations. Recent crosssectional and longitudinal studies appear to confirm a significant association between DUP and various clinical or functioning measures in successive years; the tendency is for those with a longer delay between the first psychotic episode and seeking effective treatment to show poorer clinical outcomes (Bottlender et al., 2003; Harrigan, McGorry \& Krstev, 2003; Kalla et al., 2002). The factors giving rise to a delay in beginning treatment are diverse, as is the case with treatment of some somatic illnesses, in which the very nature of the initial symptoms or the beliefs associated with the illness can determine DUP. Thus, it has been found that in psychosis, just as occurs with diabetes, an internal locus of control over health correlates with a shorter DUP (Haley, Drake, Bentall \& Lewis, 2003).

On the basis of these findings, as McGorry (1998) points out, the four main principles to be considered in carrying out early intervention in psychosis are: (a) that delay in beginning treatment is related to considerable functional deterioration, resistance to future treatment and increase in relapse rates; (b) that intensive intervention in the initial phase may reduce undesirable iatrogenic effects and aid recovery more effectively; (c) that dealing with failure in relation to initial remission of symptoms and with initial resistance to treatment, by means of psychosocial and pharmacological interventions, reduces long-term resistance to treatment, relapses and incapacity; and (d) that maintaining remission of symptoms and reducing relapses, shortening the duration of the acute symptoms of psychosis, is the post-psychosis equivalent of DUP.

With these objectives in mind, various programs of early intervention in psychosis have been launched in recent years (mainly for schizophrenic spectrum disorders), aimed at people resident in particular health service areas, with an age range of 15 to $30+$, who present prodromic symptoms or have had their first acute psychotic episode, who are without neurological or endocrinological disorders that explain the emergence of the symptoms, and who have an IQ no lower than 70. Among the best organised and well-known programs are the following: Early Psychosis Prevention and Intervention Centre (EPPIC), Melbourne, Victoria, Australia (EPPIC, 2001); Early Intervention Service (EIS), Birmingham, United Kingdom (Spencer, Birchwood \& McGovern, 2001), which includes a specific program for attention to young people (IRIS, Initiative to Reduce the Impact of Schizophrenia) (MacMillan \& Shiers, 2000); Early Treatment and Identification of Psychosis (TIPS), Norway and Denmark (Johannenssen, Larsen, McGlashan \& Vaglum, 2000; Johannessen et al., 2001); Prevention and Early Intervention Program for Psychoses (PEPP), London, Ontario, Canada (PEPP, 2000); Early Psychosis Program (EPP). Calgary, Alberta, Canada (Addington \& Addington, 2001); and The Buckingham Project (Falloon, 
2001).

Bearing in mind that, in order to achieve adequate identification and early application of treatment, it is necessary to provide sufficient information and knowledge about mental disorders and easy access to care services, and to overcome the stigma involved in seeking help at a mental health center, all of these programs include initiatives to inform and educate the general population, health professionals and teachers. Likewise, the programmes use a standard treatment protocol that combines cognitive-behavioural therapy (aimed at controlling the initial positive symptoms of the psychosis), antipsychotic medication at low doses and work with patients' families. These programs generally have a multi-professional early detection team, integrated in the healthcare network, in order to guarantee the continuity of after-care.

\section{THE SPANISH EARLY INTERVENTION PROJECT}

\section{Purpose of the project}

This research and intervention program represents an indicated primary prevention of psychoses initiative to be implemented in the public health area of Torrelavega, Cantabria (in northern Spain), with three stages: (1) the assessment of high risk personality traits; (2) the implementation of combined psychological and, where necessary, pharmacological therapies; and (3) a three-year post-treatment follow-up of cases with repeated measures.

According to the classification of risk groups made by Yung et al., the following cases are selected to enter the study (Yung \& McGorry, 1996; Yung et al., 1998):

1. Trait and state risk factors (genetic vulnerability): Individuals with non-specific neurotic symptoms, but with important severity and functional impairment, and a firstdegree relative with history of psychosis or schizotypal personality disorder. The subjects should show stable changes in mental state and on the Social Functioning Scale (SFS) (Birchwood, Smith, Cocrane, Wetton \& Copestake, 1990) for at least one month.

2. Attenuated/low grade psychotic symptoms: Individuals with at least one of the following mild symptoms: hallucinatory experiences, unusual thought content, suspiciousness, paranoid ideation, and strange behaviour. The symptoms should occur several times per week, with the mental state change lasting one week or more.

3. Transient psychotic symptoms (first episode): Individuals with moderate or severe psychotic symptoms.

The age range for the three groups is 16-30. The aim of the project is to assess the effectiveness of an intervention program for the prevention of psychosis, in the medium and long term. On the basis of empirical and theoretical work, it is hypothesized that the intervention program will reduce the probability and duration of psychotic episodes, and will improve functional adjustment and prognosis of those cases that show the disorder.

Moreover, this project represents a pilot procedure in Spain, for which there is strong demand in the public mental healthcare sector, which currently concentrates on the treatment of manifest psychotic symptoms, often at a late stage. 


\section{Study groups}

Participants are sequentially assigned to two study groups:

1. Experimental group: An estimated sample of 50 subjects per year that meet the requirements of one of the risk groups and agree to accept outpatient treatment.

2. Control group: People that fulfil the requirements of the experimental group but do not agree to outpatient treatment.

\section{Experimental design}

Because randomization cannot be used for ethical reasons, the project design is quasi-experimental. A non-equivalent group-control design will be used, with pre-treatment and several post-treatment measures (Table 1).

Analyses of Covariance and Multivariate Analyses of Covariance will mainly be performed to determine changes between groups. We shall also compare the rate of fullblown psychosis cases and admissions in the catchment area from 2003-2005 with those in previous years.

Table 1: Experimental design

\begin{tabular}{cccccc}
\cline { 3 - 5 } Groups & Assignment & \multicolumn{4}{c}{ Registration sequence } \\
\cline { 3 - 6 } & Pre-treatment & Treatment & Post-treatment ${ }_{2}$ & Post-treatment $_{n}$ \\
\hline Experimental & Non random & Observation 1 & $\mathrm{X}$ & Observation 2 & Observation $\mathrm{n}$ \\
\hline Control & Non random & Observation 1 & --- & Observation 2 & Observation $\mathrm{n}$ \\
\hline
\end{tabular}

\section{Measures}

The following instruments are used at the beginning of treatment for the assessment of individuals with attenuated psychotic symptoms and genetic vulnerability:

1. The Positive and Negative Syndrome Scale for Schizophrenia (PANSS) (Kay, Opler \& Fiszbein, 1987).

2. Frankfurt Complaint Inventory (Frankfurter Beschwerde-Fragebogen, FBF-3) (Süllwold \& Huber, 1986). This scale comprises ten subscales covering several symptom areas.

3. Early Signs System (ESS) (Birchwood et al., 1989).

4. Family Questionnaire (FQ) (Barrowclough \& Tarrier, 1992), consisting of 59 disturbing/disruptive patient behaviours that his/her relatives must rate according to frequency, amount of family burden caused by each behaviour, and the family's ability to cope with or manage them.

5. Social Functioning Scale (SFS) (Birchwood et al., 1990), which was constructed specifically to tap seven areas of functioning.

The following instruments are added for the first psychotic episode group: 
6. Structured Interview for Prodromal Syndromes (SIPS/SOPS) (McGlashan, Miller, Woods et al., 2001).

7. Personal Beliefs Questionnaire (PBIQ) (Birchwood, Mason, MacMillan \& Healy, 1993), consisting of five scales that explore beliefs on abnormal experiences.

\section{Procedure}

The mental health unit at Torrelavega (Cantabria, Spain), with a catchment area of 160,000 inhabitants, has developed a clinical protocol for early identification, assessment, and treatment of psychoses with low-dose atypical antipsychotic drugs, cognitive-behavioural therapy for adjustment and overcoming the psychosis, psychoeducation with patients and relatives after the first episode of psychosis, and cognitive therapy for positive symptoms.

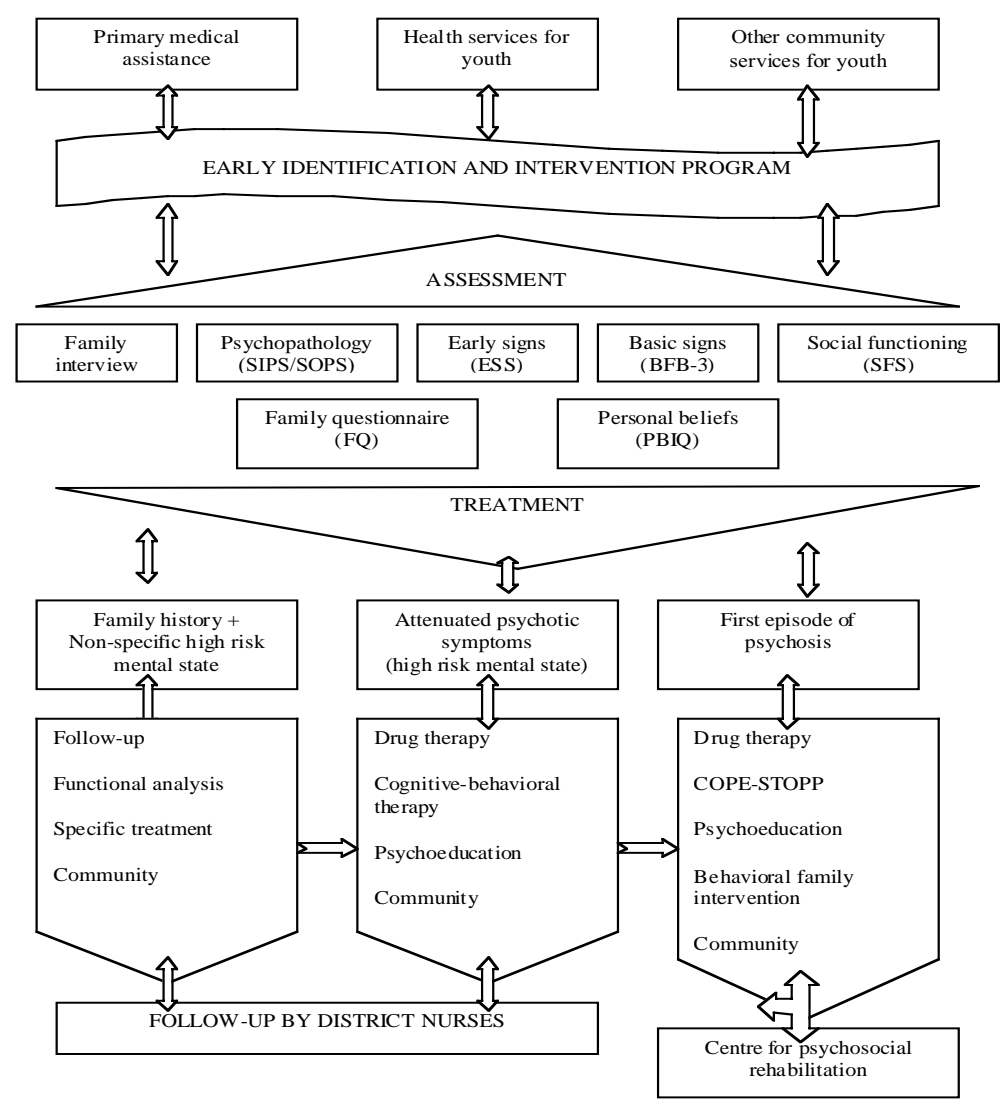

Figure 1: Protocol for early intervention in psychoses 
Cognitive behavior therapy interventions with patients involve establishing the links between their thoughts, feelings and behaviours with respect to the target symptoms, the correction of their misperceptions, irrational beliefs or reasoning biases related to those symptoms, and the promotion of alternative ways of coping with the symptoms (COPE-STOPP) (Herrmann-Doig, Maude \& Edwards, 2002; Jackson, McGorry \& Edwards, 2001). Cognitive behaviour therapy with relatives requires training them in basic communication skills, in problem-solving abilities (using a format based on Falloon, Laporta, Fadden \& Graham-Hole (1993) and on Kuipers, Leff and Lam's (1992) suggestions for expressed emotion management), and in coping skills for reducing family burden (Barrowclough \& Tarrier, 1992).

Psychoeducation involves providing information about some of the following: causes and types of psychoses and their clinical symptoms; vulnerability (protection and risk factors); antipsychotic treatments and their side effects; identification of prodromic signs; and family burden control.

All cases are referred by family doctors trained to identify early signs of psychosis, youth health services, and other community services for young people. An outline of the protocol is shown in Figure 1.

\section{REFERENCES}

Addington, J. \& Addington, D. (2001). Early intervention for psychosis: The Calgary Early Psychosis Treatment and Prevention Program. Canadian Psychiatric Association Bulletin, 33, 11-16.

Barrowclough, C. \& Tarrier, N. (1992). Families of schizophrenic patients: Cognitive behavioural intervention. London: Chapman y Hall.

Beiser, M., Erickson, D., Fleming, J.A.E. \& Iacono, W.G. (1993). Establishing the onset of psychotic illness. American Journal of Psychiatry, 150, 1349-1354.

Birchwood, M., Mason, R., MacMillan, F. \& Healy, J. (1993). Depression, demoralization and control over psychotic illness: A comparison of depressed and non-depressed patients with a chronic psychosis. Psychological Medicine, 23, 387-395.

Birchwood, M., Smith, J., Cocrane, R., Wetton, S. \& Copestake, S. (1990). The Social Functioning Scale: The development and validation of a new scale of social adjustment for use in family intervention programmes with schizophrenic patients. British Journal of Psychiatry, 157, 853859.

Birchwood, M., Smith, J., Macmillan, F., Hogg, B., Prasad, R., Harvey, C. \& Bering, S. (1989). Predicting relapse in schizophrenia: The development and implementation of an early sings monitoring system using patients and families as observers: A preliminary investigation. Psychological Medicine, 19, 649-656.

Bottlender, R., Sato, T., Jaeger, M., Wegener, U., Wittmann, J., Strauss, A. \& Moeller, H. J. (2003). The impact for the duration of untreated psychosis prior to first psychiatric admission on the 15year outcome in schizophrenia. Schizophrenia Research, 62, 37-44.

Carter, J.W. \& Mednick, S.A. (2001). Premorbid intervention: Identification of those at risk. In T.J. Miller, S.A. Mednick, T.H. McGlashan, J. Libiger \& J.O. Johannessen (Eds.), Early intervention 
in psychotic disorders. Dordrecht: Kluwer Academic. (pp. 29-45)

Cornblatt, B., Lencz, T. \& Obuchowski, M. (2002). The schizophrenia prodrome: Treatment and highrisk perspectives. Schizophrenia Research, 54, 177-186.

Cornblatt, B.A. \& Erlenmeyer-Kimling, L. (1985). Global attentional deviance as a marker of risk for schizophrenia. Journal of Abnormal Psychology, 94, 470-486.

EPPIC (2001). Case management in early psychosis: A handbook. Melbourne: Early Psychosis Prevention and Intervention Centre.

Falloon, I.R.H. (2001). Early detection and intervention through integration with primary health services: The Buckingham project. In T.J. Miller, S.A. Mednick, T.H. McGlashan, J. Libiger \& J.O. Johannessen (Eds.). Early intervention in psychotic disorders. Dordrecht: Kluwer Academic. (pp. 123-133)

Falloon, I.R.H., Laporta, M., Fadden, G. \& Graham-Hole, V. (1993). Managing stress in families: Cognitive and behavioural strategies for enhancing coping skills. London: Routledge.

Friis, S., Larsen, T.K., Melle, I., Opjordsmoen, S., Johannessen, J.O., Haahr, U., Simonsen, E., Rund, B.R., Vaglum, P. \& McGlashan, T.H. (2003). Methodological pitfalls in early detection studies: The NAPE lecture 2002. Acta Psychiatrica Scandinavica, 107, 3-9.

Häfner, H., \& Maurer, K. (2001). The prodromal phase of psychosis. In T.J. Miller, S.A. Mednick, T.H. McGlashan, J. Libiger \& J.O. Johannessen (Eds.). Early intervention in psychotic disorders. Dordrecht: Kluwer Academic. (pp. 71-100)

Häfner, H., Maurer, K., Löffler, W., An der Heiden, W., Stein, A., Könnecke, R. \& Hambrecht, M. (1999). Onset and prodromal phase as determinants of the course. In W.F. Gattaz y H. Häfner (Eds.). Search for the causes of schizophrenia. IV: Balance of the century. Berlin: Springer. (pp. 35-58)

Häfner, H., Riecher-Rössler, A., Fätkenheuer, B., Maurer, K., Meissner, S. \& Löffler, W. (1992). Interview for the Retrospective Assessment of the Onset of Schizophrenia (IRAOS). Mannheim: Central Institute of Mental Health.

Haley, C.J., Drake, R.J., Bentall, R.P. \& Lewis, S.W. (2003). Health beliefs link to duration of untreated psychosis and attitudes to later treatment in early psychosis. Social Psychiatry and Psychiatric Epidemiology, 38, 311-316.

Harrigan, S.M., McGorry, P.D. \& Krstev, H. (2003). Does treatment delay in first-episode psychosis really matter? Psychological Medicine, 33, 97-110.

Herrmann-Doig, T., Maude, D., \& Edwards, J. (2002). Systematic treatment of persistent psychosis (STOPP): A psychological approach to facilitating recovery in young people with first episode psychosis. Philadelphia, PA: Martin Dunitz.

Jackson, H. J., McGorry, P. D., \& Edwards, J. (2001). Cognitively-oriented psychotherapy for early psychosis: Theory, praxis, outcomes, and challenges. In P. W. Corrigan y D. L. Penn (Eds.), Social cognition and schizophrenia. Washington, DC: American Psychological Association. (pp. 249-284)

Johannenssen, J., Larsen, T., McGlashan, T. \& Vaglum, P. (2000). Early intervention in psychosis: The TIPS-project, a multi-centre study in Scandinavia. In B. Martindale, A. Bateman, M. Crowe, F. Margison (eds.) Psychosis: Psychological approaches and their effectiveness. London: Gaskell. (pp. 210-234)

Johannessen, J.O., Larsen, T.K., Horneland, M., Joa, I., Mardal, S., Kvebaek, R., Friis, S., Melle, I., Opjordsmoen, S., Simonsen, E., Vaglum, P. \& McGlashan, T.H. (2001). A systematized program 
to reduce duration of untreated psychosis in first episode schizophrenia. In T.J. Miller, S.A. Mednick, T.H. McGlashan, J. Libiger \& J.O. Johannessen (eds.). Early intervention in psychotic disorders. Dordrecht: Kluwer Academic. (pp. 151-166)

Jones, P.B., Rodgers, B., Murrray, R.M. \& Marmot, M.G. (1994). Child developmental risk factors for adult schizophrenia in the British 1946 birth cohort. Lancet, 344, 1398-1402.

Kalla, O., Aaltonen, J., Wahlstroem, J., Lehtinen, V., García Cabeza, I. \& González de Chávez, M. (2002). Duration of untreated psychosis and its correlates in first-episode psychosis in Finland and Spain. Acta Psychiatrica Scandinavica, 106, 265-275.

Kay, S.R., Opler, L.A. \& Fiszbein, A. (1987). The positive and negative syndrome scale (PANSS) for schizophrenia. Schizophrenia Bulletin, 13, 261-276.

Kuipers, L., Leff, J., \& Lam, D. (1992). Family work for schizophrenia: A practical guide. London: Gaskell.

Loebel, A.D., Lieberman, J.A., Alvir, J.M., Mayerhoff, D.I., Geisler, S.H. \& Szymanski, S.R. (1992). Duration of psychosis and outcome in first-episode schizophrenia. American Journal of Psychiatry, 149, 1183-1188.

MacMillan, F. \& Shiers, D. (2000). The IRIS programme. In M. Birchwood, D. Fowler \& C. Jackson (Eds.). Early intervention in psychosis: A guide to concepts, evidence and interventions. Chichester, UK: Wiley. (pp. 315-326)

Marcus, J., Hansm, S.L., Nagler, S., Auerbach, J.G., Mirsky, A.F. \& Aubrey, A. (1987). Review of the NIMH Israeli kibbutz city study and the Jerusalem infant development study. Schizophrenia Bulletin, 13, 425-438.

McGlashan, T.H. (1998). Early detection and intervention of schizophrenia: Rationale and research. British Journal of Psychiatry, 172 (Suppl.33), 3-6.

McGlashan, T.H., Miller, T.J. \& Woods, S.W. (2001). Pre-onset detection and intervention research in schizophrenia psychoses: Current estimates of benefit and risk. Schizophrenia Bulletin, 27, 563-570.

McGlashan, T.H., Miller, T.J., Woods, S.W., Rosen, J.L., Hoffman, R.E. \& Davidson, L. (2001). Structured Interview for Prodromal Syndromes. New Haven, CT: PRIME Research Clinic. Yale School of Medicine.

McGorry, P. (1998). Preventive strategies in early psychosis: Verging on reality. British Journal of Psychiatry, 172 (Suppl. 33), 1-2.

McGorry, P.D. (1998). "A stitch in time"... the scope for preventive strategies in early psychosis. European Archives of Psychiatry and Clinical Neuroscience, 248, 22-31.

McGorry, P.D. \& Jackson, H.J. (Eds.). (1999). The recognition and management of early psychosis: A preventive approach. Cambridge, UK: Cambridge University Pess.

Mirsky, A.F., Kugelmass, S., Ingraham, L.J., Frenkel, E. \& Nathan, M. (1995). Overview and summary: Twenty-five-year followup of high risk children. Schizophrenia Bulletin, 21, 227-239.

Moscarelli, M. (1994). Health and economic evaluation in schizophrenia: Implications for health policies. Acta Psychiatrica Scandinavica, 84 (Suppl. 382), 84-88.

Mrazek, P.J. \& Haggerty, R.J. (1994). Reducing risks for mental disorders: Frontiers for preventive intervention research. Washington, DC: National Academy Press.

Olin, S.C.S., Mednick, S.A., Cannon, T., Jacobsen, B., Parnas, J., Schulsinger, F. \& Schulsinger, H. (1998). School teacher ratings predictive of psychiatric outcome 25 years later. British Journal of Psychiatry, 172 (Suppl.33), 7-13. 
PEPP. (2000). Prevention and early intervention program for psychosis: Screening, assessment, and treatment manuals. London, Ontario: London Health Sciences Centre.

Pincus, H.A., Davis, W.W. \& McQueen, L.E. (1999). Subthreshold mental disorders: A review and synthesis of studies on minor depression and other 'brand names'. British Journal of Psychiatry, $174,288-296$.

Rapoport, J.L., Giedd, J.N., Blumenthal, J.A., Hamburger, S.N.J., Fernández, T., Nicolson, R., Bedwell, J., Lenane, M., Zijdenbos, A., Paus, T. \& Evans, A. (1999). Progressive cortical change during adolescence in childhood onset schizophrenia: A longitudinal MRI study. Archives of General Psychiatry, 56, 649-654.

Salokangas, R.K.R., Heinimaa, M., Ilonen, T., Suomela, T., Korkelia, J., Plathin, M., Ristkari, T., Huttunen, J., Hietala, J., Syvälahti, E., \& McGlashan, T.H. (2001). Epidemiology of prodrome in familial schizophrenia: Description of the DEEP project. In T.J. Miller, S.A. Mednick, T. H. McGlashan, J. Libiger \& J. O. Johannessen (Eds.), Early intervention in psychotic disorders. Dordrecht: Kluwer Academic. (pp. 47-69)

Spencer, E., Birchwood, M., \& McGovern, D. (2001). Management of first-episode psychosis. Advances in Psychiatric Treatment, 7, 133-142.

Squires-Wheeler, E., Friedman, D. \& Erlenmeyer-Kimling, L. (1993). A longitudinal study relating P3 amplitude to schizophrenia spectrum disorders and to global personality functioning. Biological Psychiatry, 33, 774-785.

Süllwold, L. \& Huber, G. (1986). Schizophrene basisstörungen. Berlin: Springer Verlag.

Vallina Fernández, O., \& Lemos Giráldez, S. (2001). Tratamientos psicológicos eficaces para la esquizofrenia. Psicothema, 13, 345-364.

Watt, N.F., Anthony, E.J., Wynne, L.C. \& Rolf, J.E. (1984). Children at risk for schizophrenia: A longitudinal perspective. New York: Cambridge University Press.

Yung, A.R. \& McGorry, P.D. (1996). The prodromal phase of first episode psychosis: Past and current conceptualizations. Schizophrenia Bulletin, 22, 353-370.

Yung, A.R., McGorry, P.D. \& McFarlane, C.A. (1996). Monitoring and care of young people at incipient risk of psychosis. Schizophrenia Bulletin, 22, 283-303.

Yung, A.R., Phillips, L.J., McGorry, P.D., McFarlane, C.A., Francey, S., Harrigan, S., Patton, G.C. \& Jackson, H.J. (1998). Prediction of psychosis: A step towards indicated prevention of schizophrenia. British Journal of Psychiatry, 172 (Suppl. 33), 14-20.

Received November 3, 2003 Final acceptance November 27, 2003 